\title{
Adie Pupil as the Initial Presentation of Localized En Coup de Sabre Scleroderma
}

RONALD M. LAXER, MDCM, FRCPC, Division of Rheumatology, The Hospital for Sick Children, and Department of Paediatrics, University of Toronto; STEVEN MILLER, MDCM, FRCPC, Division of Neurology, The Hospital for Sick Children, and Department of Paediatrics, University of Toronto; ELENA POPE, MD, MSc, Section of Dermatology, The Hospital for Sick Children, and Department of Paediatrics, University of Toronto, Toronto, Ontario, Canada. Address correspondence to Dr. R.M. Laxer, Hospital for Sick Children, Rheumatology, 555 University Ave., Toronto, Ontario M5G 1X8, Canada.

E-mail: ronald.laxer@sickkids.ca. J Rheumatol 2017;44:1096-7; doi:10.3899/jrheum.161442. Research Ethics Board approval was not required for this report, in accordance with the policy of The Hospital for Sick Children, Toronto, Ontario, Canada, regarding case reports. Written informed consent was obtained.

Ocular involvement in facial morphea can take multiple forms, including changes in the eyelashes and eyelids, ptosis, uveitis, episcleritis, paralytic strabismus, orbital myositis, enophthalmos, pseudopapilledema, refractive errors, and dry eye; mydriasis has been reported occasionally ${ }^{1,2}$. Adie pupil has been recognized as a rare association ${ }^{3}$. Awareness of these associations is important to possibly prevent ocular complications.
A 15-year-old girl was noted to have a left pupil larger than the right at 9 years of age. Ophthalmological evaluation showed arrested contraction consistent with Adie pupil, a tonically dilated pupil that reacts slowly to light and more robustly to accommodation. Magnetic resonance scan done at age 11 years (Figure 1A, courtesy of Dr. Helen Branson) showed a nonenhancing lesion in the left frontal lobe white matter consistent with gliosis and subcutaneous tissue loss in
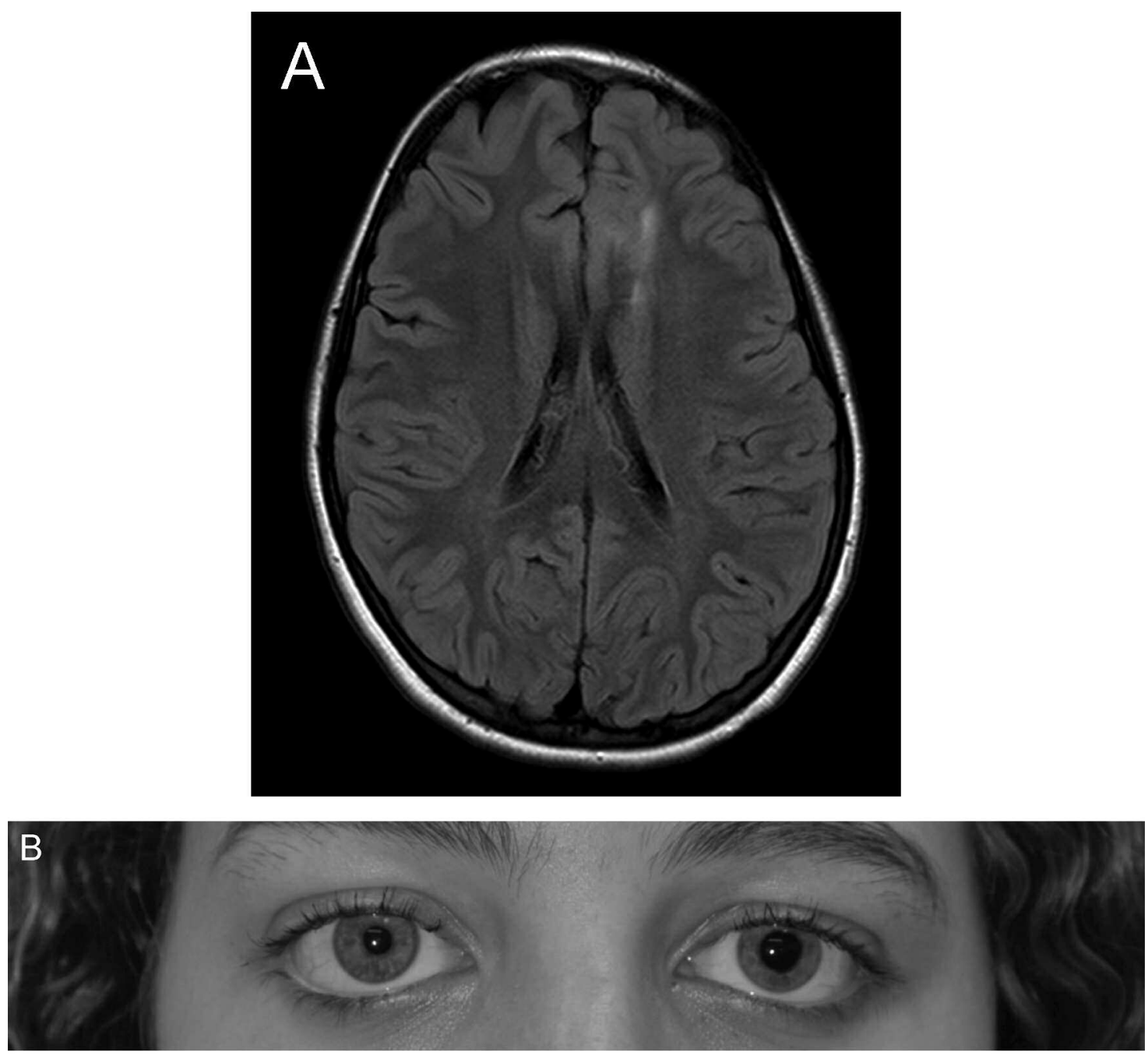

Figure 1. A. Magnetic resonance imaging of the brain of an 11-year-old girl (courtesy of Dr. Helen Branson) showing a nonenhancing lesion in the left frontal lobe white matter and subcutaneous tissue loss in the left frontal region. B. Patient at age 14 years showing pupillary irregularity, with left pupil larger than right. 
the left frontal region. A computed tomography angiogram was normal. At the age of 14 years, in addition to the pupillary abnormality (Figure 1B), slight asymmetry of the face was noted with mild deviation of the chin toward the left, loss of creases, and skin folds suggesting mild hemiatrophy. A linear area of thick skin and decreased subcutaneous tissue, all covered by hair, was noted extending from the left forehead toward the vortex of her scalp (Figure 2A). The left side of her palate was smaller than her right and dental abnormalities were observed with a gap between the left central and lateral incisors (Figure 2B). There has been no progression of Adie pupil or development of other neurologic or autonomic problems to the present time (age $15 \mathrm{yrs}$ ). She was diagnosed with localized en coup de sabre scleroderma (ECDS $)^{4}$.

Clinicians must recognize the association of ECDS lesions with Adie pupil because it may lead to earlier diagnosis of facial localized scleroderma, allowing for intervention and prevention of cutaneous and extracutaneous disease progression.

\section{REFERENCES}

1. Zannin ME, Martini G, Athreya BH, Russo R, Higgins G, Vittadello F, et al; Juvenile Scleroderma Working Group of the Pediatric Rheumatology European Society (PRES). Ocular involvement in children with localised scleroderma: a multi-centre study. Br J Ophthalmol 2007;91:1311-4.

2. Bucher F, Fricke J, Neugebauer A, Cursiefen C, Heindl LM. Ophthalmological manifestations of Parry-Romberg syndrome. Surv Ophthalmol 2016;61:693-701.

3. Aynaci FM, Sen Y, Erdöl H, Ahmetoğlu A, Elmas R. Parry-Romberg syndrome associated with Adie's pupil and radiologic findings. Pediatr Neurol 2001;25:416-8.

4. Laxer RM, Zulian F. Localized scleroderma. Curr Opin Rheumatol 2006;18:606-13.
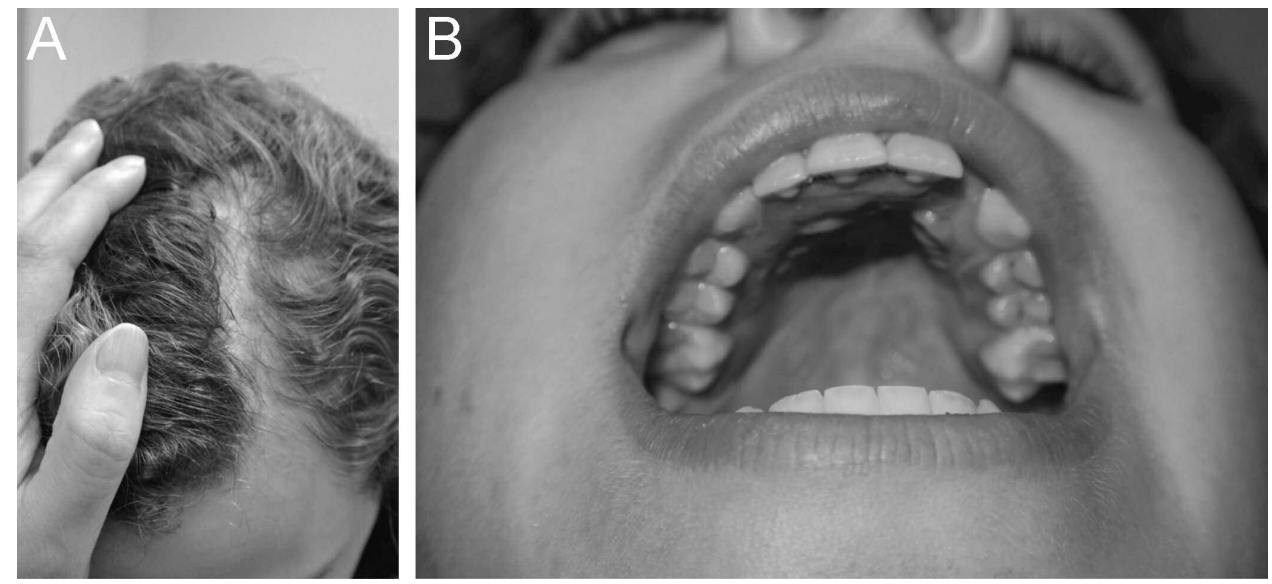

Figure 2. Patient at age 14 with en coup de sabre localized scleroderma showing (A) a linear area of thick skin and decreased subcutaneous tissue extending from the left forehead toward the vortex of her scalp, and (B) asymmetry of the palate (left side smaller) and dental abnormalities, with a gap between the left central and lateral incisors. 